\title{
Ensure Symmetrical Traffic Flow, to prevent the Dropping of Response Packet by the Firewall, on the Active-Active Data Centers
}

\author{
Irwan Piesessa \\ Computer Science Department, BINUS Graduate Program - Master of Computer Science, Bina Nusantara University, \\ Jakarta, Indonesia 11480 \\ E-mail:irwan.piesessa@gmail.com \\ Benfano Soewito \\ Computer Science Department, BINUS Graduate Program - Master of Computer Science, Bina Nusantara University, \\ Jakarta, Indonesia 11480 \\ E-mail: bsoewito@binus.edu
}

Received: 23 February 2018; Accepted: 11 May 2018; Published: 08 June 2018

\begin{abstract}
This paper illustrates the problem in the Active-Active Data Centers of an organization, where response traffic from the destination server is dropped by the firewall because the initial traffic from the client departs from another firewall in different Data Center (asymmetric traffic). This problem can be solved by two proposed solutions, namely the implementation of the BGP Community attributes and OSPF over GRE tunnel. The case study also compares both proposed solutions in terms of recovery time, packet loss, ICMP response time and TCP three-way handshake time for HTTP connection.
\end{abstract}

Index Terms - Asymmetric routing, symmetric routing, Border Gateway Protocol (BGP), Open Shortest Path First (OSPF), Generic Routing Encapusulation (GRE).

\section{INTRODUCTION}

Routing is the process of finding the best path from the source system to the destination system on a network [1]. Asymmetric routing is a problem that often occurs in organizations that have two data centers to connect to the internet or to other networks through different firewalls. In asymmetric routing, the first TCP packet departs from the source with a syn flag (Synchronize) [2] to destination using one path and uses a different path when the response, TCP packet from the destination server (with the syn-ack flag) is sent back to the source [3]. The response packets from the server (for example, TCP (Transmission Control Protocol) syn-ack packet)) will be dropped by the firewall on the return path. This is because this firewall does not have a connection or session table for the related response packets. The connection or session table for clients that transmits initial packets (for example, TCP syn packet), resides on the firewall that is on the packet depart path.

Some solutions have been offered in terms of firewall configuration, for example, is to disable the tcp-check feature [4]. However, this will increase the security risk on the network. This paper offers a solution from the side of the routing protocol without reducing the level of network security (without disabling security features on the firewall).

An organization in Indonesia ("Organization A") have a Primary and Secondary Data Center that connected their Indonesian Head Office to their overseas Regional Data Center. Their overall IGP (Interior Gateway Protocol) Diagram can be seen in fig. 1 .

"Organization A" uses OSPF as its IGP routing protocol where OSPF (Open Shortest Path First) is currently the most commonly used IGP protocols [5]. OSPF uses link-state or technology based on the SPF (Shortest Path First) to build and calculate the shortest route/path to all known destinations [6].

For EGP (Exterior Gateway Protocol), they use BGP (Border Gateway Protocol). BGP has many attributes or parameters that can be used to determine the best route to a network. An Administrator can select policies that match his/her organization's needs using BGP. With these advantages BGP was designed in the early 1990s [7] is de-facto used as an inter-domain routing protocol on the internet [8]. "Organization A" BGP network topology can be seen in fig. 2 .

To load balance the links to the Primary Data Center (DC-A) and the Secondary Data Center (DC-B), traffic from the Head Office were divided as below:

- Intranet and all web application traffic to Regional DC, will enter DC-B first, then it will be forwarded to DC-A and will go to Regional DC through DC-A firewall.

- Email and other traffic to Regional DC will go to DC-A first, then it will be forwarded to DC-A via DC-A Firewall. 
To achieve the above routing policy, the BGP localpreference attribute is used. Local-preference is a BGP attribute that belongs to a well known discretionary type that is included in each router update from one router to another router within the same Autonomous System [9].
If two or more routes have the same network prefix in the BGP table, then if the routes have same weight value, a route with higher local-preference value will be selected [10].

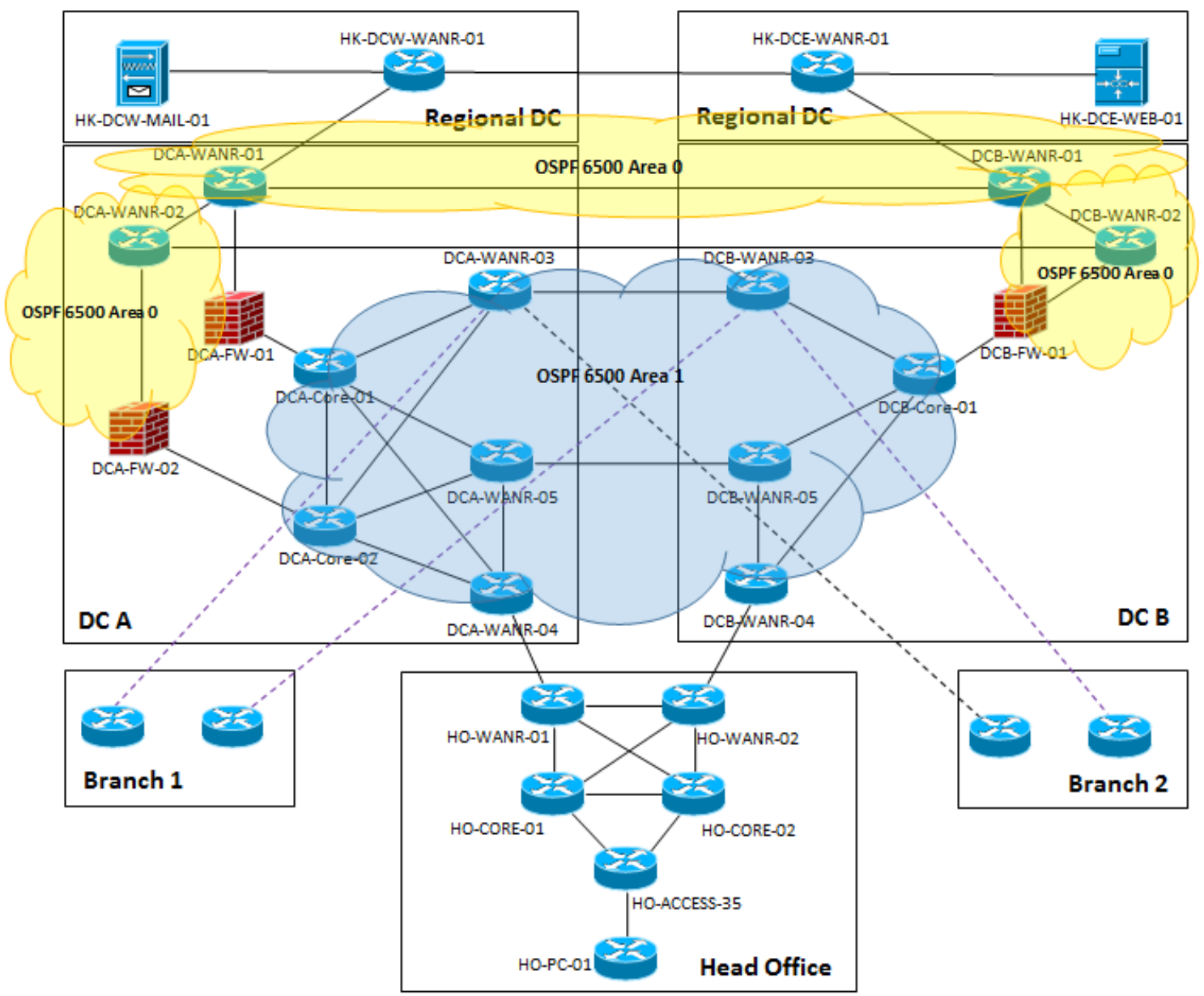

Fig.1. OSPF Topology

In "Organization A" head office network, on WAN (Wide Area Network) router that's connected to the DC-A, network prefix for Intranet and web application servers are set to have a local-preference value of 150 . And other network prefixes that include the email server will have local-preference value 200. On Head Office WAN router that's connected to DC-B, the intranet and web application server network prefix are set to have a localpreference value of 200, and other network prefix set to 100. The local-preference configuration of the WAN routers in the network head office can be seen in table 1 .

Table 1. BGP Local-preference Setting

\begin{tabular}{|c|c|c|}
\hline \multicolumn{3}{|c|}{ BGP Local Preference Value } \\
\hline Router & $\begin{array}{c}\text { Intranet+Web } \\
\text { Servers Network } \\
\text { Prefixes }\end{array}$ & $\begin{array}{c}\text { Mail \& Other } \\
\text { Network Prefixes }\end{array}$ \\
\hline HO-WANR-01 & 150 & 200 \\
\hline HO-WANR-02 & 200 & 100 \\
\hline
\end{tabular}

Based on BGP local-preference attribute on table 1, the traffic flow is shown in fig. 3 .
The "Organization A" routing policy also states that traffic from the Head Office must flow through their dedicated Data Center routers (IE DCB-WANR-04, DCB-WANR-05, DCA-WANR-04 and DCA-WANR-05) to distribute their WAN routers workload in DC-A and DC-B. To achieve this policy, these routers are reconfigured the Administrative Distance (AD) values of their BGP routing protocols. AD is a feature that routers use to select the best path when there are two or more different routes to the same destination of two different routing protocols. AD defines the reliability of routing protocols. Each routing protocol has different priority values [11].

DCB-WANR-04，DCB-WANR-05，DCA-WANR-04 and DCA-WANR-05 are configured with AD 105 for internal BGP, so BGP routes will be preferred over OSPF that has default AD, which is 110 [12]. They allocate other domestic WAN routers (IE DCA-WAN-03 and DCB-WANR-03) for their branch traffic.

To speed up the routing convergence time, BGP keepalive and holdtime setting on dedicated Data Center WAN routers for Head Office are reconfigured. BGP 
holdtime is the time value set by the router to determine whether the bgp peer is live or not If within a specified holdtime the router does not receive a keepalive message from its peer, then it will declare that the BGP peer is dead [13]. Usually holdtime time is three times the keepalive time [14].

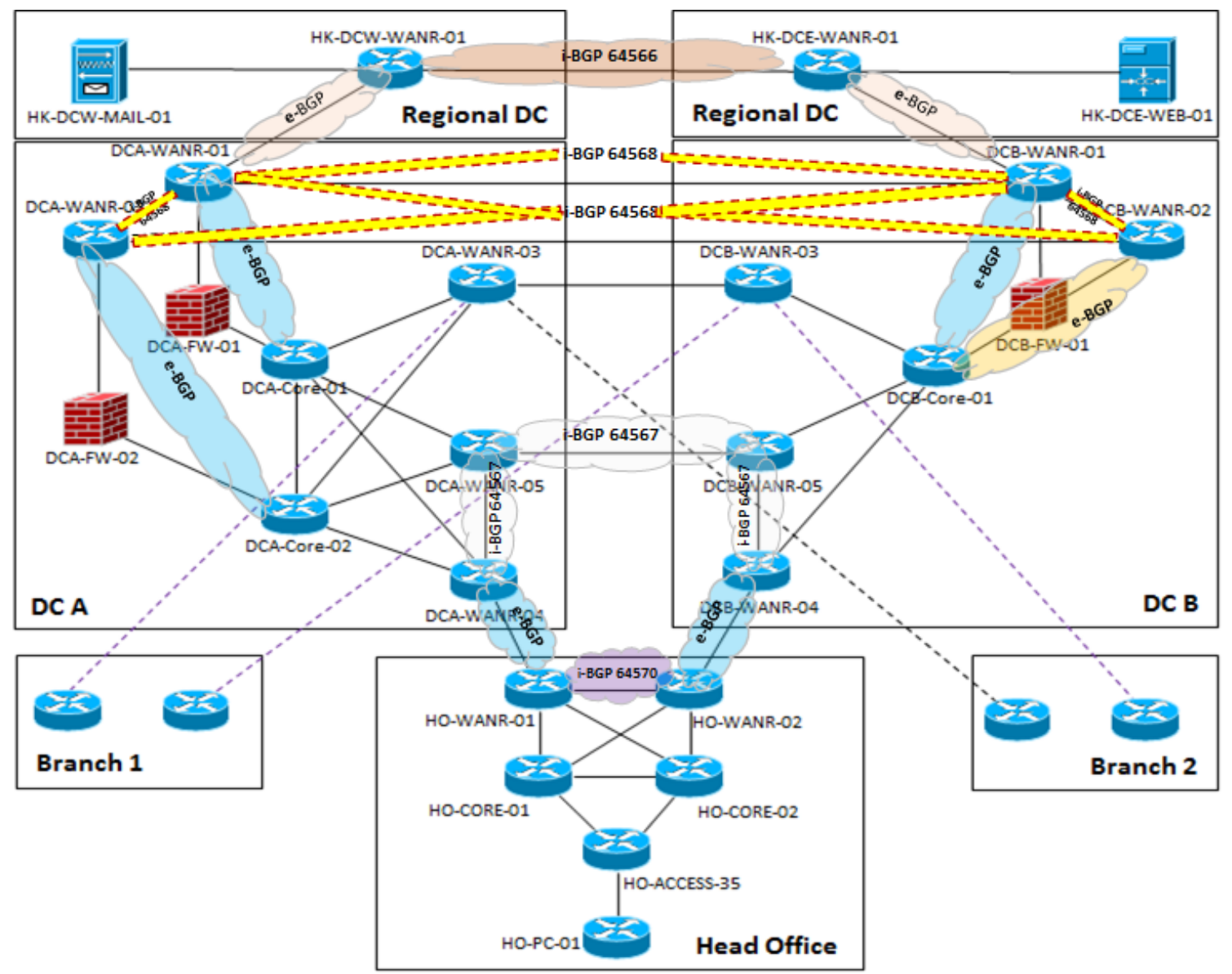

Fig.2. BGP Topology

DCB-WANR-04，DCB-WANR-05，DCA-WANR-04 and DCA-WANR-05 are reconfigured with a 5-second BGP keepalive time and a 10-second BGP holdtime. With this configuration, the BGP speaker router will send a keepalive message to the BGP peer in every 5 seconds. If within 10 seconds there is no BGP keepalive message received by the router, it will be declared that BGP peer is down, and all BGP routes received from the peer will be removed from the BGP table.

We simulate "Organization A" networks, including the Head Office network in Indonesia, their Primary and Secondary Data Centers (DC-A and DC-B) and Overseas Data Centers, with the same topology, the same router vendor and network operating systems, the same IGP and EGP routing protocols (OSPF and BGP), the same OSPF Areas and network type, and the same BGP routing policies (including the same BGP attributes, timers and Administrative Distance) as well as the same traffic flow for intranet, email and other applications.

To confirm the network traffic flow according to fig. 3, below is the traceroute output from the client PC at Head Office (HO-PC-01) to the intranet web server:

HO-PC-01\#traceroute 10.15.3.100

Tracing the route to 10.15 .3 .100
1 10.23.35.1 $32 \mathrm{msec} 12 \mathrm{msec} 32 \mathrm{msec}$

$210.23 .33 .564 \mathrm{msec} 36 \mathrm{msec} 12 \mathrm{msec}$

$310.23 .33 .18124 \mathrm{msec} 56 \mathrm{msec} 176 \mathrm{msec} \leftarrow$ HO-WANR-02

$410.23 .255 .5276 \mathrm{msec} 184 \mathrm{msec} 136 \mathrm{msec} \leftarrow$ DCB-WANR-04

5 10.19.0.54 $140 \mathrm{msec} 140 \mathrm{msec} 132 \mathrm{msec} \leftarrow$ DCB-WANR-05

6 10.18.255.1 $168 \mathrm{msec} 144 \mathrm{msec} 100 \mathrm{msec} \leftarrow$ DCA-WANR-05

7 10.18.0.13 $188 \mathrm{msec} 144 \mathrm{msec} 108 \mathrm{msec} \leftarrow$ DCA-Core- 01

8 10.18.0.38 $112 \mathrm{msec} 108 \mathrm{msec} 152 \mathrm{msec}$

$910.18 .0 .26196 \mathrm{msec} 160 \mathrm{msec} 172 \mathrm{msec}$

10 10.19.2.2 $164 \mathrm{msec} 156 \mathrm{msec} 160 \mathrm{msec}$

11 10.15.0.13 $236 \mathrm{msec} 180 \mathrm{msec} 172 \mathrm{msec}$ $1210.15 .0 .18220 \mathrm{msec} 212 \mathrm{msec} 216 \mathrm{msec} \leftarrow$ Intranet Web Server

\section{ASYMMETRIC Routing PROBLEM}

The problem occurs when the inter-DC link (DCA-DCB link between DCA-WANR-05 - DCB-WANR-05) down. Traffic to the intranet server from the Head Office network will remain through the Head Office - DC-B WAN link. Then, because of their routing policy, traffic 
from Head Office will not flow through their dedicated branches WAN routers (IE DCB-WANR-03 and DCAWANR-03), but will be forwarded by DCB-WANR-05 to the DC-B Core Router and go to the intranet server in
Regional DC via DC-B firewall. And because of their routing policy as well, the response traffic will return to Head Office through DC-A via DC-A firewall. This is explained in the traffic flow diagram in fig. 4.

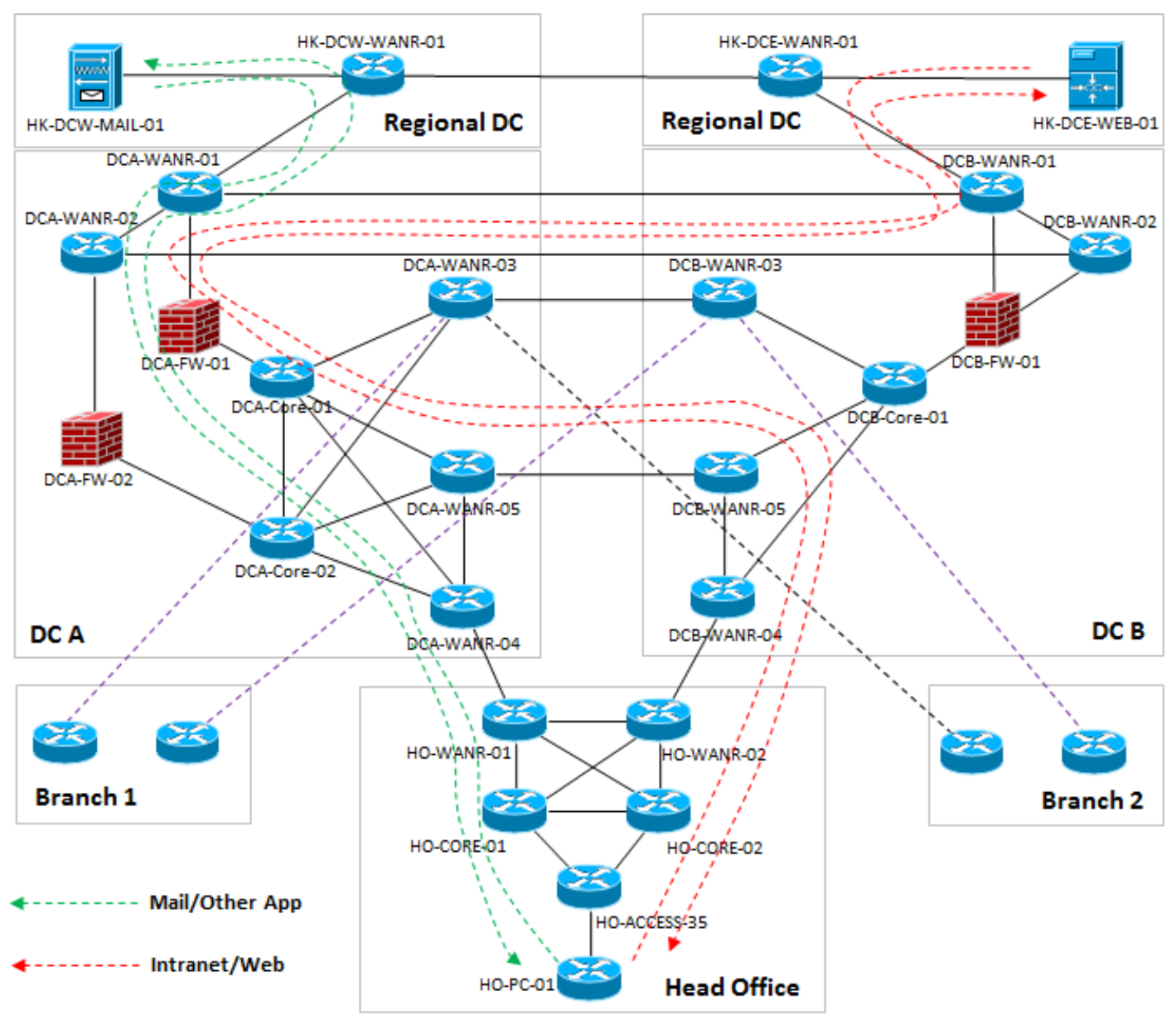

Fig.3. Routing Policy for Network Traffic Flow

Because of this, the connection to the intranet server can not be done. This can be seen from the ping results that run from the client $\mathrm{PC}$ to the intranet server before and after the inter-DC link is disconnected as follows:

HO-PC-01\#ping 10.15.3.100 rep 200

Type escape sequence to abort.

Sending 200, 100-byte ICMP Echos to

10.15.3.100, timeout is 2

seconds: !!!!!!!!!!!!!!!!!!!!!!!!!! ...UUU...UUUUU UUUUUUUUUUUUUUU

\section{PROPOSED SOLUTIONS}

After analyzing their network architecture and routing policy, then to solve this case, we propose two solutions as follows:

- Using the BGP community

- Using OSPF peering over GRE (Generic Routing Encapsulation) tunnel

\section{A. Using the BGP Community}

Community is an optional transitive attribute of the BGP. BGP routers can use this attribute to determine whether to accept or not and determine the priority level of the received BGP route [15].

We propose to use BGP communities in the BGP networks to detect whether the inter-DC link between DCA-WANR-05 and DCB-WANR-05 is in normal condition (up) or not (down). We configured the DCAWANR-05 router to add the BGP community attribute to all routes sent to the DC-B (DCB-WANR-05) router and will be advertised to DCB-WANR-04 and HO-WANR02. Both WAN routers at Head Office (IE HO-WANR-01 and HO-WANR-02) will know about the conditions of inter-DC link based on the routes they receive from DC-B. If there is a community string on the route, then the interDC link is in normal condition. And if it does not exist, then they will state that the inter-DC link is down.

We use 64567: 1000 as a BGP community value on the DCA-WANR-05 router to route table that will be advertised to the DCB-WANR-05 router. 


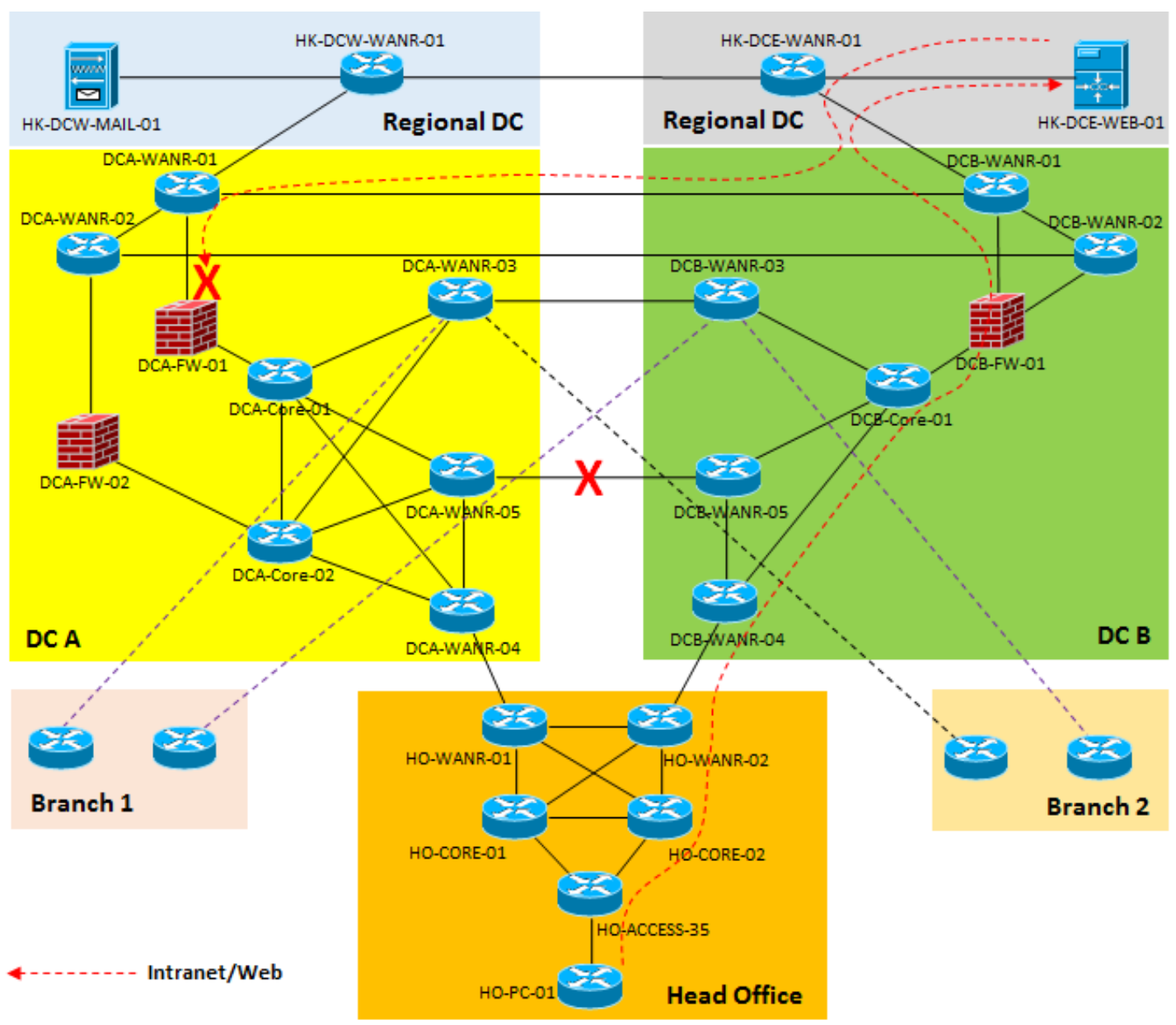

Fig.4. Asymmetric Routing Problem

In $\mathrm{HO}-\mathrm{WANR}-02$ router, we set the BGP rule that if the route with the intranet server network prefix is received from DCB-WANR-04 and has a BGP community with value 64567: 1000, we will set the route to have BGP local-preference with value 200. For other routes we will set the route to have a BGP localpreference BGP with value 100. This BGP route table will be installed in the router's routing table and advertised to the HO-WANR-01 router. The BGP rule on the HO-WANR-02 router at Head Office network can be seen in table 2 .

Table 2. BGP rule on HO-WANR-02

\begin{tabular}{|c|c|c|}
\hline \multicolumn{3}{|c|}{ Router HO-WANR-02 } \\
\hline \multicolumn{2}{|c|}{ If Match (and) } & Then Set \\
\hline $\begin{array}{c}\text { Network = Intranet } \\
\text { Servers Prefix }\end{array}$ & $\begin{array}{c}\text { Community }= \\
64567: 1000\end{array}$ & local-preference $=200$ \\
\hline \multicolumn{2}{|c|}{ Network = Other Prefixes } & local-preference $=100$ \\
\hline
\end{tabular}

Verification of the BGP best route to the Intranet server on both of the WAN routers in the Head Office, can be seen in fig. 5 and 6 . For route received from the DCB-WANR-04 router, which informs that for network intranet server prefix $(10.15 .3 .100 / 32)$ that have community BGP 64567: 1000, it will go to Regional DC via router HO-WANR-02 (10.23.1.4) then forwarded to DCB-WANR-04 (10.23.255.5).
Paths: (3 available, best \#1, table Default-IP-Routing-Table, RIB-failure(17)) Flag: 0x9E0

Advertised to update-groups:

1

64567, (received \& used)

10.23.255.5 (metric 1) from 10.23.1.4 (10.23.1.4)

Origin incomplete, metric 1000 , localpref 200 , valid, internal, best Community: 64567:1000

Fig.5. BGP route table on HO-WANR-01 router

BGP routing table entry for 10.15.3.100/32, version 153

Paths: (2 available, best \#1, table Default-IP-Routing-Table)

Advertised to update-groups:

2

64567

10.23.255.5 from 10.23.255.5 (10.19.1.5)

Origin incomplete, metric 1000 , localpref 200 , valid, external, best Community: 64567:1000

Fig.6. BGP route table on HO-WANR-01 router

With the combination of local-preference and the BGP community attributes, if the inter-DC link between DCAWANR-05 and DCB-WANR-05 is down, HO-WANR-02 will not receive the intranet server prefix from the DC-B router that has the BGP community with a value of 64567 : 1000. Please note that we have created a BGP rule in the HO-WANR-02 router, that the route to be given the highest local-preference (200), is the route for the intranet 
server network prefix (10.15.3.100/32) and has the BGP community attribute 64567: 1000 (using AND operation). Because of this, the router will delete the previous route, and will install the intranet server prefix originating from the DC-A via the HO-WANR-01 router. Then, all HO WAN routers will direct traffic to the intranet server to the DC-A via HO-WANR-01 (10.23.1.3) and then through DCA-WANR-04 (10.23.255.1).

Once the BGP community solution configuration has been implemented in the simulator, we simulate by disconnecting the inter-DC link between DCA-WANR05 and DCB-WANR-05. The BGP route table for the intranet server prefix (10.15.3.100) on the HO-WANR-01 and HO-WANR-02 routers after the inter-DC link between DCA-WANR-05 and DCB-WANR-05 is disconnected, can be seen in fig. 7 and 8 .

BGP routing table entry for $10.15 .3 .100 / 32$, version 107

Paths: (2 available, best \#1, table Default-IP-Routing-Table)

Flag: 0x9C0

Advertised to update-groups:|

2

64567

10.23.255.1 from 10.23.255.1 (10.18.1.5)

Origin incomplete, metric 1000 , localpref 150 , valid, external, best

Fig.7. BGP route table on HO-WANR-01 after inter-DC link down
HO-WANR-02\#sh ip bgp 10.15.3.100

BGP routing table entry for $10.15 .3 .100 / 32$, version 157

Paths: (2 available, best \#2, table Default-IP-Routing-Table, RIB-failure(17))

Flag: 0x820

Advertised to update-groups:

1

64567, (received \& used)

10.23.255.1 (metric 2) from 10.23.1.3 (10.23.1.3)

Origin incomplete, metric 1000, localpref 150, valid, internal, best

Fig.8. BGP route table on HO-WANR-02 after inter-DC link down

The network flow to the intranet server after inter-DC link between DCA-WANR-05 and DCB-WANR-05 is disconnected and the BGP community configuration is implemented can be seen in fig. 9 .

Output of the ping command on HO-PC-01 to the intranet web server which confirms that traffic has recovered after inter-DC connection between DCAWANR-05 and DCB-WANR-05 disconnected is shown below:

HO-PC-01\#ping 10.15.3.100 rep 20

Sending 20, 100-byte ICMP Echos to 10.15.3.100, timeout is 2 seconds:

!!!!!!!!!! ...!!!!!!!!!

Success rate is 85 percent $(17 / 20)$, roundtrip $\mathrm{min} / \mathrm{avg} / \mathrm{max}=180 / 243 / 328 \mathrm{~ms}$ HO-PC-01\#

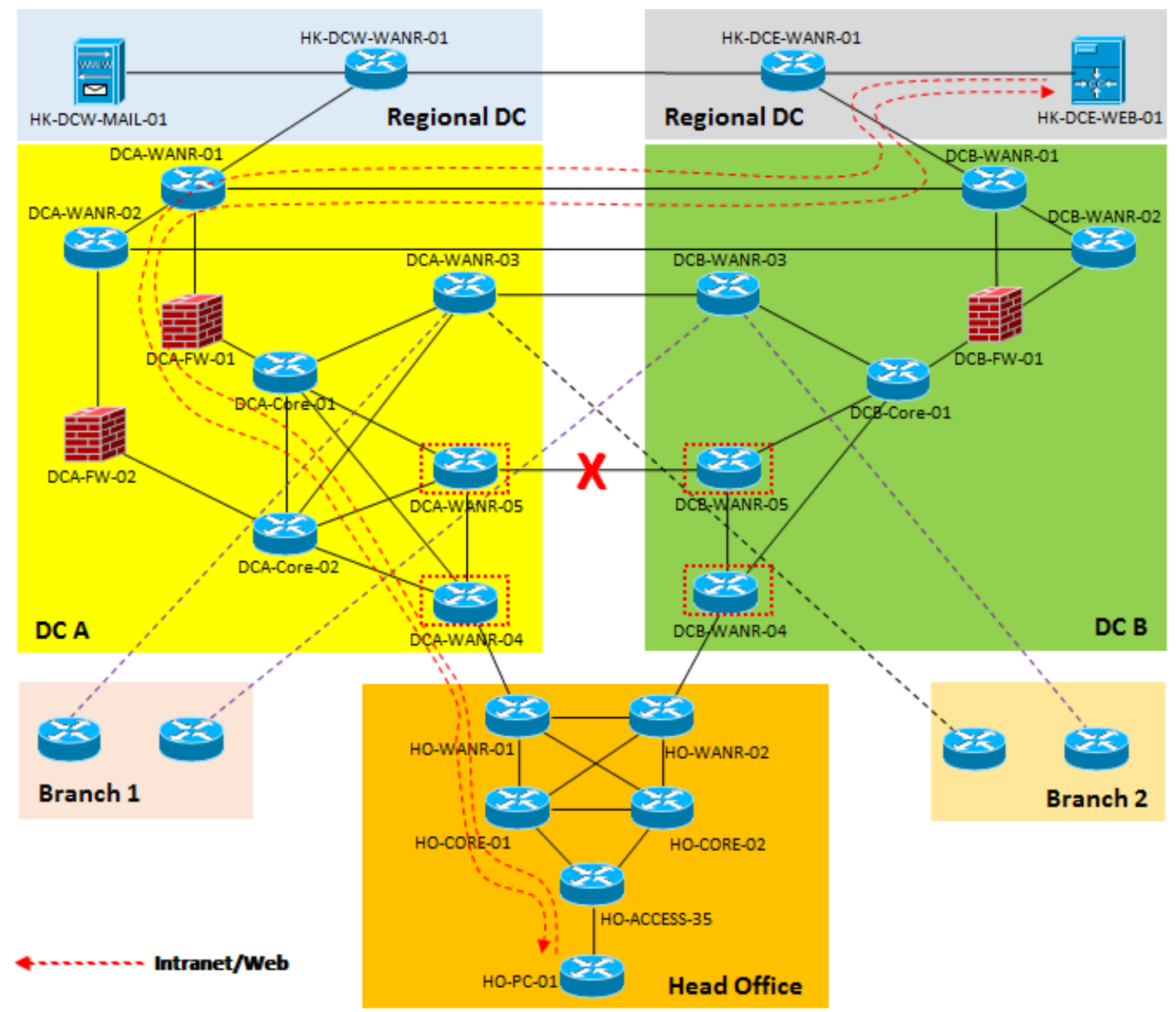

Fig.9. Traffic flow from Head Office to Intranet Server after the Inter-DC link down 
There are $3 \mathrm{icmp}$ (Internet Control Message Protocol) echo-request packets that get no reply during the route transition when the inter-DC link is disconnected. The traceroute output below from the Head Office PC to the intranet server confirms that network traffic to the intranet server is now via DC-A:

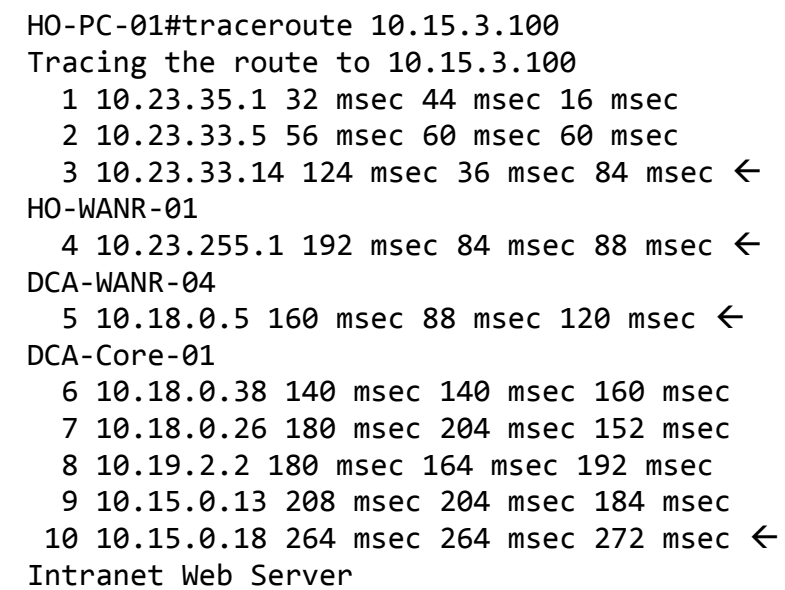

Based on the BGP route table, ping, http (Hypertext Transfer Protocol) access and traceroute commands from our network simulations, we can state that BGP community solutions can solve asymmetric routing problems on "Organization A" networks.

\section{B. Using OSPF over GRE Tunnel Solution}

We propose an alternative solution, based on the OSPF routing protocol that used as IGP in DC-A and DC-B. The idea is to utilize the inter-DC link between DCA-
WANR-03 and DCB-WANR-03 in accordance with company policy allocated for use only by the branch network. Because in DCA-WANR-04, DCA-WANR-05, DCB-WANR-04 and DCB-WANR-05, BGP internal routing protocol (with $\mathrm{AD} 105$ ) is preferred over OSPF (AD 110), we introduce the route to regional DC in OSPF that will be promoted and installed in the DCB-WANR05 routing table when the BGP peering session between DCA-WANR-05 and DCB-WANR-05 is disconnected. OSPF peering will be established on top of the new GRE Tunnel. The GRE is a protocol used to encapsulate a packet over other protocols that run over the network layer [16]. GRE runs over a virtual point-to-point connection. The GRE packet structure can be seen in fig. 10 [17]:

\begin{tabular}{|c|}
\hline Delivery Header \\
\hline GRE Header \\
\hline Payload Packet \\
\hline
\end{tabular}

Fig.10. GRE Packet Structure

We propose to use the new loopback IP address as the source and destination address. This IP address will not be advertised to IGP to prevent routing loops. The new loopback IP address will be routed through static routes or other routing protocols that have a lower $\mathrm{AD}$ than OSPF. This OSPF peering will always be available both when the inter-DC link between DCA-WANR-05 and DCB-WANR-05 is normal or disconnected (down).

OSPF peering diagram running on the GRE Tunnel we propose can be seen in fig. 11 and 12 .

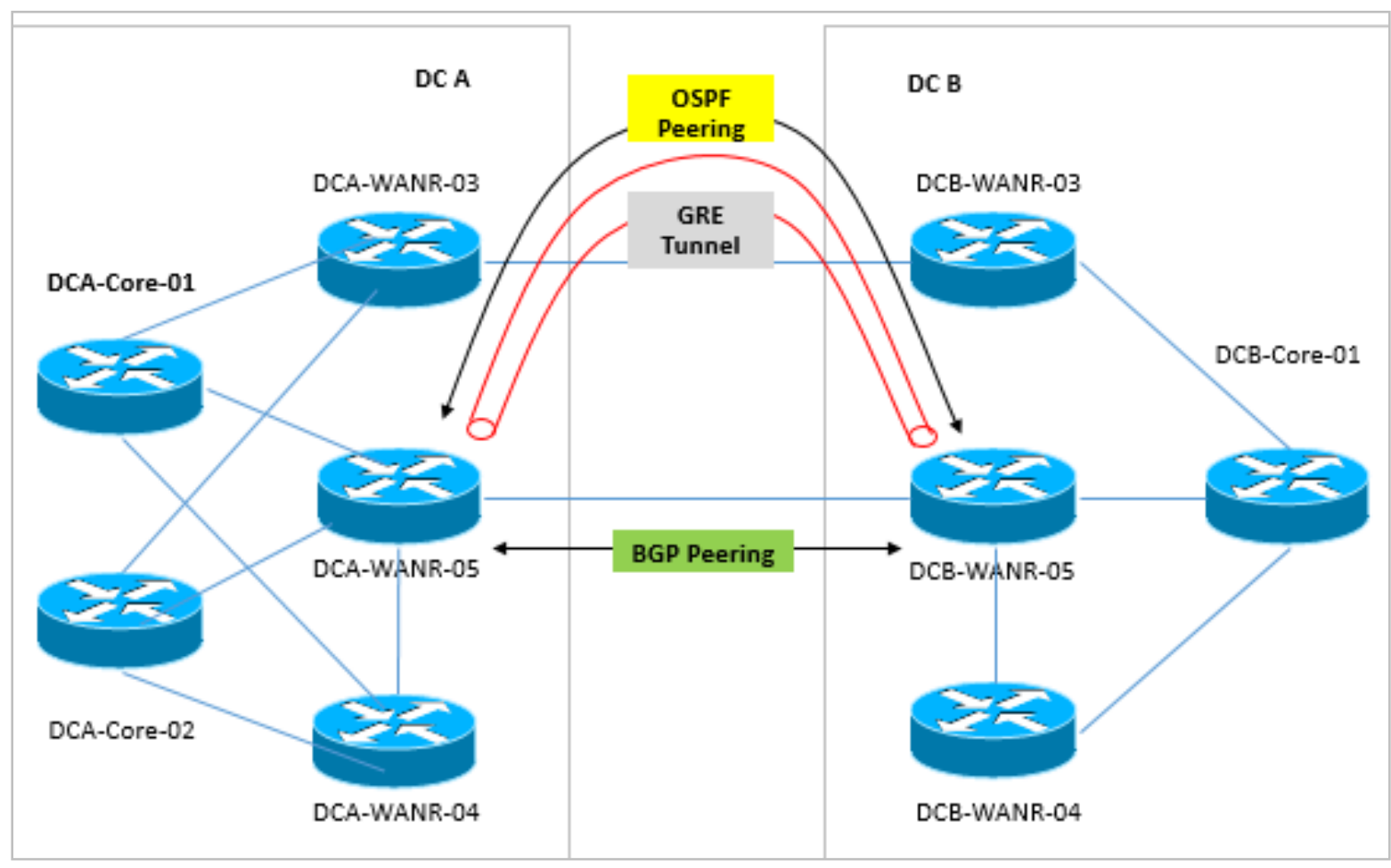

Fig.11. OSPF over GRE tunnel with inter-DC link between DCA-WANR-05 and DCB-WANR-05 in Normal Condition 


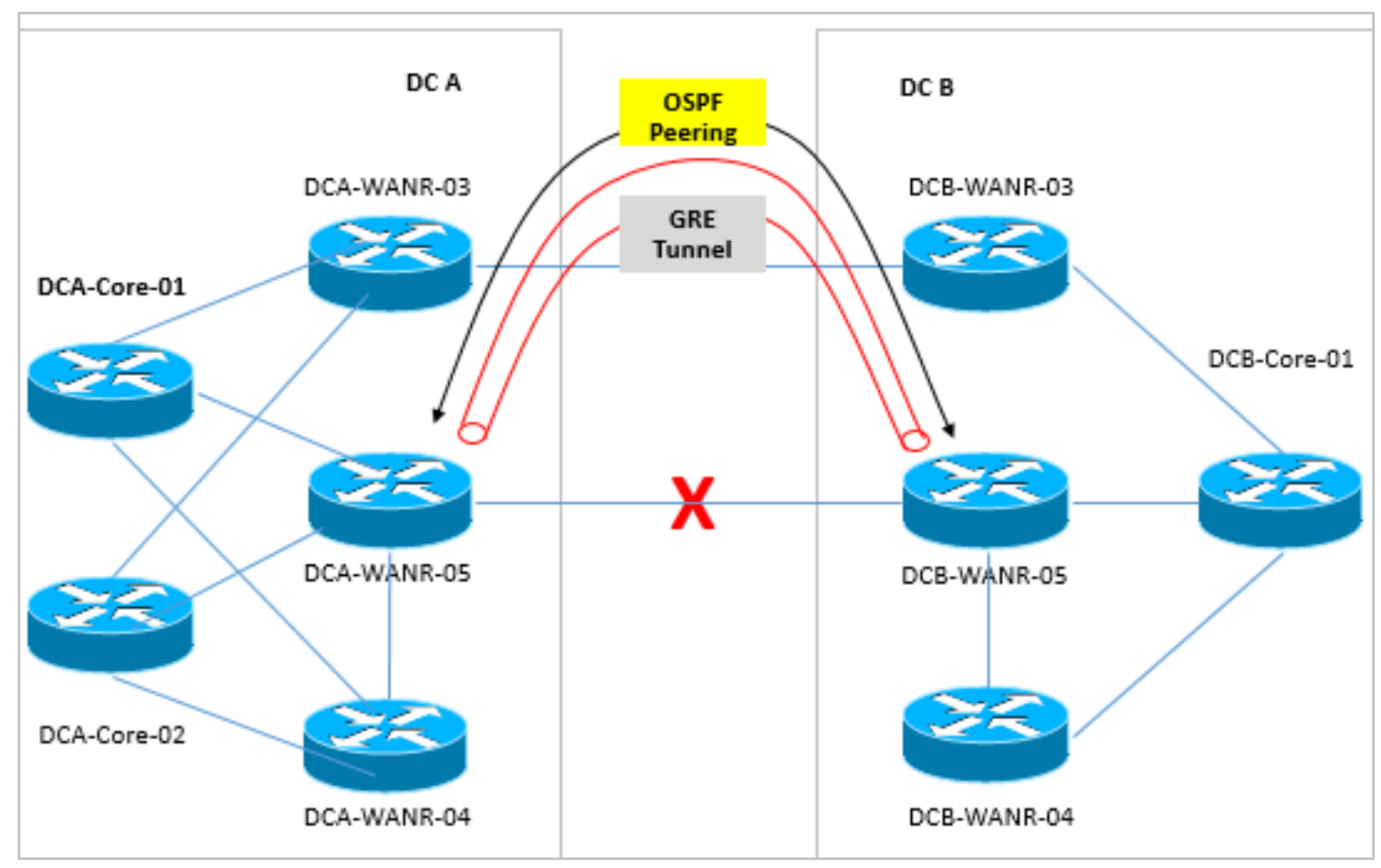

Fig.12. OSPF Peering over GRE tunnel is still available when the inter-DC link between DCA-WANR-05 and DCB-WANR-05 is disconnected.

After we define the configuration of OSPF peering over GRE Tunnel that needs to be applied to the network, then we implement it in the simulator. OSPF peering session information between DCA-WANR-05 and DCBWANR-05 over GRE Tunnel is shown in fig. 13 and 14.

\section{Neighbor 10.18.1.6, interface address 10.18.254.1 In the area 1 via interface Tunnel 100 \\ Neighbor priority is 0 , State is FULL, 6 state changes $\mathrm{DR}$ is $0.0 .0 .0 \mathrm{BDR}$ is 0.0 .0 .0 \\ Options is $0 \times 52$ \\ LLS Options is $0 \times 1$ (LR)}

Fig.13. OSPF peers over GRE Tunnel (Interface Tunnel 100) on DCAWANR-05 router (peering to DCB-WANR-05)

Once the OSPF peering over GRE Tunnel configuration has been implemented in the simulator, we simulate by disconnecting the inter-DC link between DCA-WANR-05 and DCB-WANR-05. The network traffic flow that going to intranet server after the inter-DC link between DCA-WANR-05 and DCB-WANR-05 disconnected can be seen in fig. 15 .

Output of ping and telnet to TCP port 80 on HO-PC-01 to the intranet web server which ensures that the network traffic is successfully recovered after the inter-DC link between DCA-WANR-05 and DCB-WANR-05 is disconnected, can be seen below:

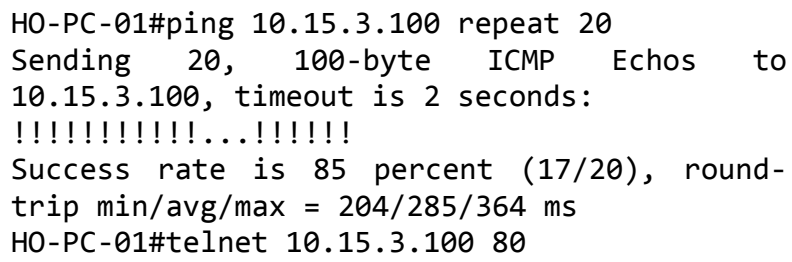

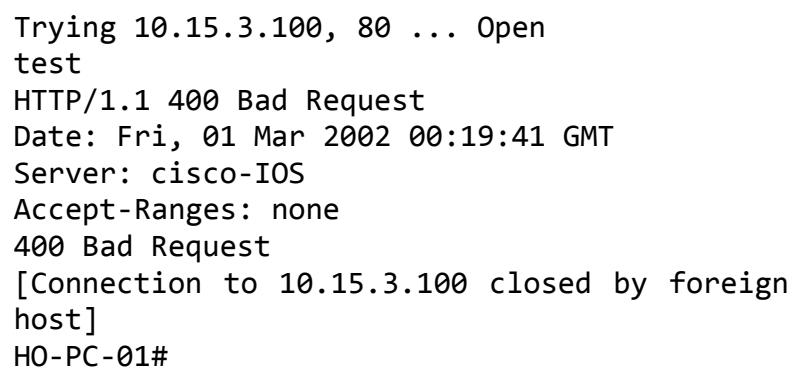

The output of the traceroute of the PC in the Head Office below ensures that now the network traffic flowing to the intranet server will enter DC-B first and then redirected to the DC-A via a new GRE tunnel between DCA-WANR-05 and DCB-WANR-05:

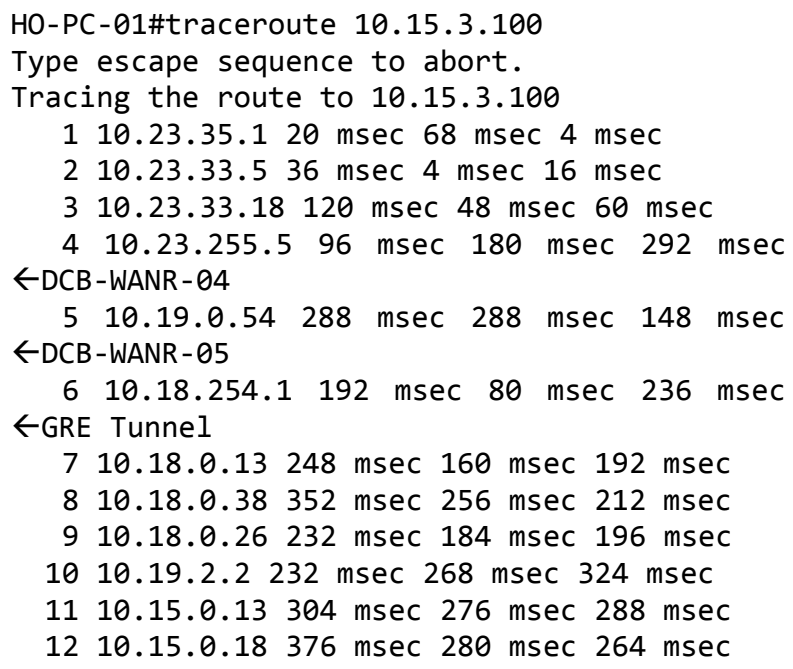

Based on the simulation, we can argue that the OSPF 


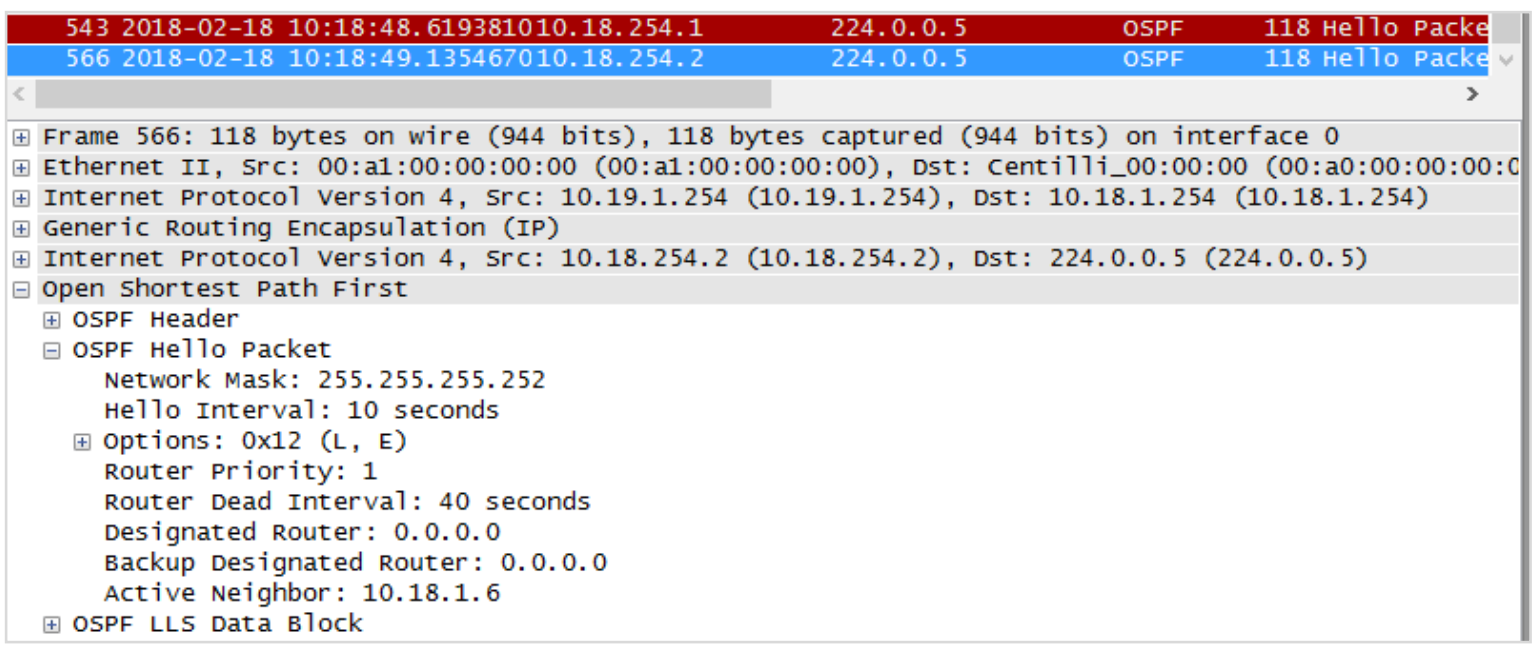

Fig.14. Network Packet Analyzer reveals OSPF Hello packet over GRE Tunnel

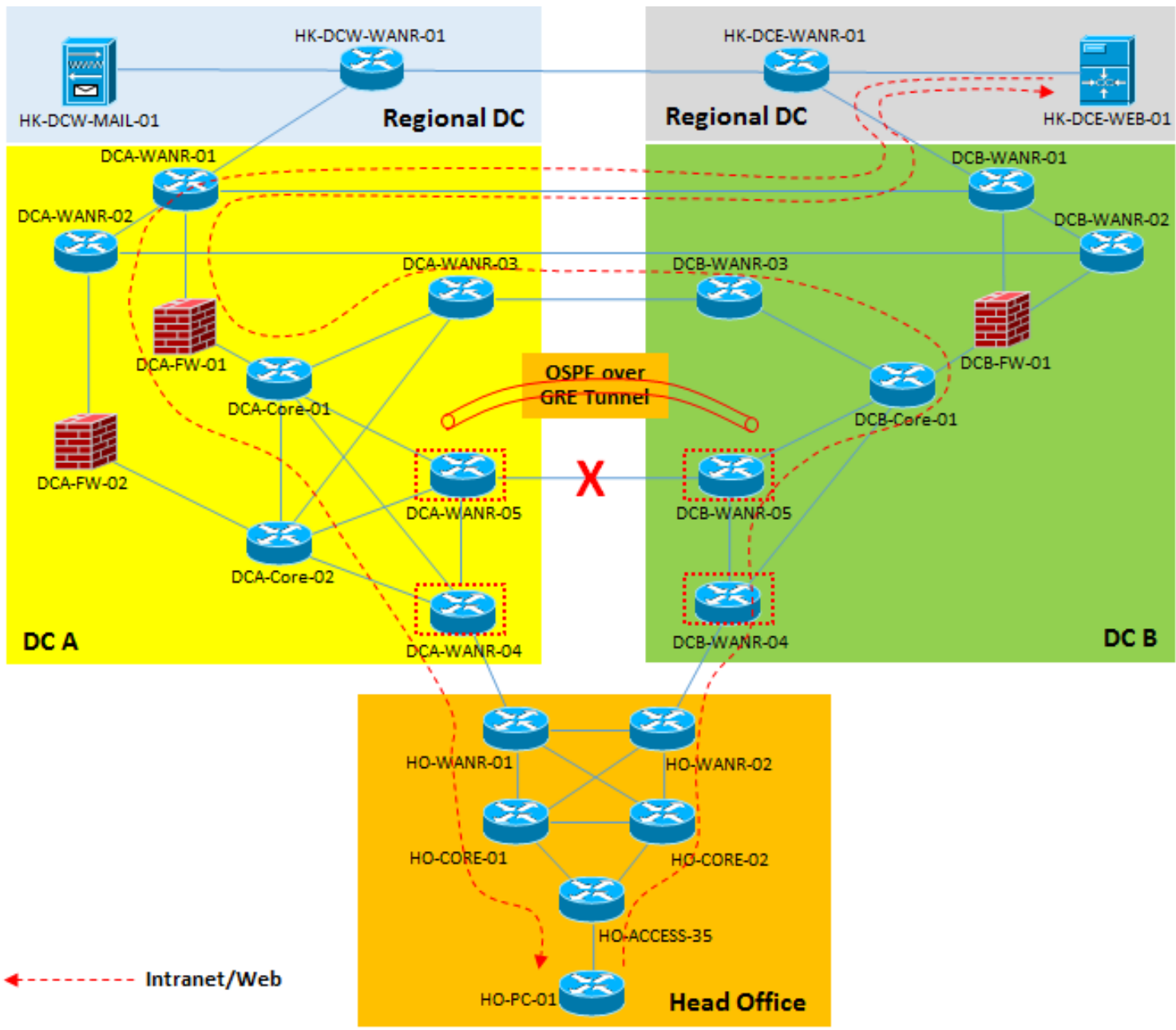

Fig.15. Traffic flow from Head Office to Intranet Server after the Inter-DC link disconnected and OSPF over GRE Tunnel solution applied

\section{Evaluation OF THE PROPOSED SOLUTIONS}

We use the following parameters to evaluate both the solutions we offer:
1. Recovery time.

2. Packet loss.

3. ICMP echo-reply time.

4. TCP three-way handshake time for HTTP. 


\section{A. Recovery Time}

Recovery time is how fast the flowing traffic can be recovered after the inter-DC link between DCA-WANR05 to DCB-WANR-05 is disconnected. In every test, we record icmp echo packet time from client $\mathrm{PC}$ that does not get the reply from the intranet server (IEx). (IEx) is sent by the $\mathrm{PC}$ client to the intranet server before the inter-DC link between DCA-WANR-05 and DCBWANR-05 is disconnected. We also record icmp echo packet time sent by the client $\mathrm{PC}$ to the intranet server after the traffic can be restored either by the BGP Community or OSPF over the GRE Tunnel solution (IEy). The recovery time (RT) can be calculated by using the following formula.

$$
\mathrm{RT}=I E \mathrm{y}-I E \mathrm{x}
$$

ICMP echo is an ICMP packet with type 8 [18].

We tested both solutions 30 times. The result of all tests in terms of recovery time is shown in table 3 .

Table 3. Recovery Time

\begin{tabular}{|c|c|c|}
\hline Test & $\begin{array}{l}\text { BGP Community } \\
\text { (second) (a) }\end{array}$ & $\begin{array}{l}\text { OSPF over GRE Tunnel } \\
\text { (second) (b) }\end{array}$ \\
\hline 1 & 6.02035 & 6.14471 \\
\hline 2 & 5.98595 & 5.97645 \\
\hline 3 & 5.99584 & 6.25964 \\
\hline 4 & 6.01240 & 5.99296 \\
\hline 5 & 6.00371 & 6.18292 \\
\hline 6 & 6.01631 & 6.02487 \\
\hline 7 & 6.00475 & 6.14596 \\
\hline 8 & 6.01561 & 6.01575 \\
\hline 9 & 6.00210 & 6.00673 \\
\hline 10 & 6.00322 & 6.55538 \\
\hline 11 & 6.02087 & 5.89943 \\
\hline 12 & 6.01559 & 5.98652 \\
\hline 13 & 6.02839 & 6.18638 \\
\hline 14 & 5.63500 & 6.36581 \\
\hline 15 & 5.98416 & 5.95480 \\
\hline 16 & 6.00923 & 6.22222 \\
\hline 17 & 6.05173 & 5.99795 \\
\hline 18 & 6.00117 & 6.66694 \\
\hline 19 & 5.98637 & 5.99063 \\
\hline 20 & 6.12940 & 6.17405 \\
\hline 21 & 5.55354 & 6.34709 \\
\hline 22 & 6.15758 & 6.14246 \\
\hline 23 & 6.01870 & 6.30851 \\
\hline 24 & 5.98481 & 6.96698 \\
\hline 25 & 5.99764 & 5.99581 \\
\hline 26 & 5.93496 & 6.02806 \\
\hline 27 & 5.99675 & 6.36990 \\
\hline 28 & 5.99669 & 6.02636 \\
\hline 29 & 5.90589 & 5.99147 \\
\hline 30 & 6.09340 & 6.16391 \\
\hline Average & 5.98540 & 6.16969 \\
\hline \multicolumn{2}{|c|}{ Difference (b-a) } & 0.18428 \\
\hline \multicolumn{2}{|c|}{ Percentage of difference (b-a) } & $3.0 \%$ \\
\hline
\end{tabular}

Comparison of recovery time of each test for both solutions can be seen in fig. 16.

We can see that based on table 3 and fig. 16, when we use the BGP community solution, the average recovery time is $184.3 \mathrm{~ms}$ faster than OSPF over GRE tunnel.

According to the author's analysis, recovery time in
BGP Community solutions is faster, because the router that decides to turn traffic to PDC after getting information about inter-DC interruption is the HOWANR-01 router that has two BGP routes to go to the intranet server (10.15.3.100/32), which is through SDC via HO-WANR-02 router and through PDC via PDCWANR-04 router. The best route is through SDC. When the inter-DC link is disconnected, the HO-WANR-01 router will receive this information and will delete the route to the intranet server via SDC. Then route to the intranet server via PDC will be the best BGP route and promoted to the routing table of the router.

So the processes performed by the HO-WANR-01 router are:

1. Delete BGP route to the intranet server 10.15.3.100 via SDC

2. Making the BGP route to the intranet server 10.15.3.100 via PDC (pre-existing in the BGP table as an alternate route) becomes the best BGP route to 10.15.3.100

3. Promote BGP route to the intranet server 10.15.3.100 to the routing table of the router.

As for the OSPF over GRE Tunnel solution, when the SDC-WANR-05 router receives disconnected information on PDC-SDC inter-DC links, the following are the following processes:

1. Perform a recalculation process to determine the best route to the intranet server 10.15.3.100 (partial process spfQ)

2. Added a new OSPF route to the intranet server 10.15.3.100 to the OSPF database.

3. Delete the OSPF database for the route to the intranet server 10.15.3.100 via PDC-SDC inter-DC link.

4. Promote the new OSPF route to the intranet server to the routing table of the router.

More processes are being performed on OSPF over the GRE Tunnel solution to find the best route after PDCSDC inter-DC link interrupted. This makes recovery time on OSPF over GRE Tunnel solution longer than BGP Community solution.

The author's analysis is based on the process log of old route deletion and new route installation, either for OSPF over GRE Tunnel solution or BGP Community solution. The following is a $\log$ of the process of deleting and adding routes to the intranet server of the SDC-WANR-05 router when the PDC-SDC inter-DC link is disconnected (using OSPF over GRE Tunnel solution):

\footnotetext{
*Mar 1 01:17:02.579: RT: del 10.15.3.100/32 via 10.18.255.1, bgp metric [105/1000]

*Mar 1 01:17:02.579: RT: delete subnet route to $10 \cdot 15 \cdot 3 \cdot 100 / 32$

*Mar 1 01:17:02.579: RT: NET-RED

$10.15 \cdot 3.100 / 32$

*Mar 1 01:17:02.863: OSPF process partial

spfQ LSA id 10.15.3.100: mask 255.255.255.255, type 5 adv_rtr 10.18.1.1, age 629, seq
} 
0x80000006 (Area dummy area)

*Mar 1 01:17:02.863: OSPF: Start partial processing Type 5 External LSA 10.15.3.100, mask 255.255.255.255, adv 10.18.1.1, age 629, seq 0x80000006, metric 1000, metric-type 2 * Mar 1 01:17:02.867: Add better path to LSA ID 10.15.3.100, gateway 10.18.254.1, dist 1000

*Mar 1 01:17:02.867: Add path: next-hop 10.18.254.1, interface Tunnel100

*Mar 1 01:17:02.867: OSPF: delete lsa id 10.15.3.100, type 5, adv rtr 10.18.1.1 from delete list

*Mar 1 01:17:02.867: network update dest_addr 10.15.3.100 mask 255.255.255.255 gateway 10.18.254.1

*Mar $101: 17: 02.871:$ RT: SET_LAST_RDB for $10.15 .3 .100 / 32$

NEW rdb: via 10.18.254.1

* Mar 1 01:17:02.871: RT: add 10.15.3.100/32

via 10.18.254.1, ospf metric [110/1000]

*Mar 1 01:17:02.871: RT: NET-RED $10.15 \cdot 3 \cdot 100 / 32$

*Mar 1 01:17:02.875: Add External Route to 10.15 .3 .100 . Metric: 1000, Next Hop: 10.18 .254 .1

Based on the logs above, seen in the routing table of the old route deletion process and the new route promotion / installation takes 296 milliseconds (time in the last line of the logs minus the time on the first line).

The following are the process logs of old route deletion and new route installation when using BGP Community solution on the HO-WANR-01 router when PDC-SDC inter-DC link is disconnected:

\section{HO-WANR-01\#}

Mar 1 03:07:01.659: $\operatorname{BGP}(0): 10.23 .1 .4 \mathrm{rCV}$ UPDATE about 10.15.3.100/32 -- withdrawn

Mar 1 03:07:01.667: BGP(0): Revise route installing 1 of 1 routes for 10.15.3.100/32 -> 10.23.255.1(main) to main IP table

Mar 1 03:07:01.667: RT: closer admin distance for 10.15.3.100, flushing 1 routes Mar 1 03:07:01.667: RT: NET-RED $10.15 \cdot 3.100 / 32$

Mar 1 03:07:01.667: RT: SET_LAST_RDB for $10.15 .3 .100 / 32$

NEW rdb: via 10.23.255.1

Mar 1 03:07:01.671: RT: add 10.15.3.100/32 via 10.23.255.1, bgp metric [20/0]

Mar 1 03:07:01.671: RT: NET-RED $10.15 \cdot 3.100 / 32$

$\operatorname{Mar} 103: 07: 01.675: \operatorname{BGP}(0): 10.23 .1 .4$ NEXT_HOP part 1 net $10.15 .3 .100 / 32$, next 10.23 .255 .1

HO-WANR-01\#

Based on the above logs, we can see that the old route deletion process and the promotion / installation of new routes in the BGP table take 12 milliseconds (faster than when using OSPF over GRE solution).

\section{B. Packet Loss}

In this test, packet loss is an ICMP echo packet (from client PC) that does not get icmp echo-reply packet (from intranet server) during the transition period. Number of packet loss after we tested two solutions we offered 30 times can be seen in table 4 .

The comparison of number of ICMP-echo packet that does not get ICMP echo-reply packet for each test of both solutions can be seen in fig. 17.

Based on table 4 and fig. 17, we can see that when we use the BGP community solution, the average number of icmp echo-request packets that get no reply is $23.1 \%$ lower than the OSPF over GRE Tunnel solution.

Table 4. Number of ICMP Echo Packet That Does Not Get a Reply

\begin{tabular}{|c|c|c|}
\hline Test & $\begin{array}{l}\text { BGP Community } \\
\text { Solution } \\
\text { (a) }\end{array}$ & $\begin{array}{l}\text { OSPF over } \\
\text { GRE Tunnel } \\
\text { (b) }\end{array}$ \\
\hline 1 & 3 & 4 \\
\hline 2 & 3 & 3 \\
\hline 3 & 3 & 4 \\
\hline 4 & 3 & 3 \\
\hline 5 & 3 & 3 \\
\hline 6 & 3 & 3 \\
\hline 7 & 3 & 4 \\
\hline 8 & 3 & 3 \\
\hline 9 & 3 & 3 \\
\hline 10 & 3 & 6 \\
\hline 11 & 3 & 4 \\
\hline 12 & 3 & 3 \\
\hline 13 & 3 & 4 \\
\hline 14 & 3 & 5 \\
\hline 15 & 3 & 3 \\
\hline 16 & 3 & 4 \\
\hline 17 & 3 & 3 \\
\hline 18 & 3 & 7 \\
\hline 19 & 3 & 3 \\
\hline 20 & 3 & 3 \\
\hline 21 & 3 & 5 \\
\hline 22 & 3 & 4 \\
\hline 23 & 3 & 5 \\
\hline 24 & 3 & 4 \\
\hline 25 & 3 & 3 \\
\hline 26 & 3 & 4 \\
\hline 27 & 3 & 6 \\
\hline 28 & 3 & 3 \\
\hline 29 & 3 & 4 \\
\hline 30 & 3 & 4 \\
\hline Average & 3 & 3.9 \\
\hline \multicolumn{2}{|c|}{ Difference (b-a) } & 0.9 \\
\hline \multicolumn{2}{|c|}{ Percentage of difference (b-a) } & $23.1 \%$ \\
\hline
\end{tabular}

The number of icmp echo-request packets that get no response is closely related to the recovery time value of each solution. So in accordance with the author's analysis on the recovery time test results, the number of ICMPecho-request packets that get no response in the BGP Community solution were fewer, as the number of processes performed to remove and promote new routes to the intranet server in the routing table on the SDCWANR-05 (on the OSPF over GRE Tunnel solution) is much more than the process done by the HO-WANR-01 router in the BGP Community solution.

\section{ICMP Echo-request Reply Time (The Ping Delay)}

This parameter is measured from the average ICMP 
echo-reply packet time as the response packet for the ICMP echo packet sent by the client PC in the Head Office to the intranet server at Regional DC after the traffic is restored. In every test, we sent the icmp echo packet 100 times from the PC client to the intranet server after the traffic returned to normal while the inter-DC link remained disconnected. ICMP echo reply is an ICMP packet with type 0 .

The average ping delay from the client $\mathrm{PC}$ to the intranet server when we use both solutions after the interDC link is disconnected can be seen in table 5 .

Table 5. Average of ICMP Echo-reply Time

\begin{tabular}{|c|c|c|}
\hline Test & $\begin{array}{c}\text { BGP Community } \\
\text { Solutions } \\
\text { (miliseconds) }\end{array}$ & $\begin{array}{c}\text { OSPF over GRE } \\
\text { Tunnel } \\
\text { (miliseconds) }\end{array}$ \\
\hline 1 & 247 & 394 \\
\hline 2 & 230 & 312 \\
\hline 3 & 285 & 373 \\
\hline 4 & 240 & 381 \\
\hline 5 & 258 & 351 \\
\hline 6 & 229 & 320 \\
\hline 7 & 234 & 311 \\
\hline 8 & 232 & 286 \\
\hline 9 & 244 & 293 \\
\hline 10 & 225 & 333 \\
\hline 11 & 224 & 303 \\
\hline 12 & 231 & 309 \\
\hline 13 & 228 & 323 \\
\hline 14 & 228 & 297 \\
\hline 15 & 226 & 301 \\
\hline 16 & 224 & 324 \\
\hline 17 & 226 & 299 \\
\hline 18 & 229 & 298 \\
\hline 19 & 240 & 323 \\
\hline 20 & 230 & 330 \\
\hline 21 & 244 & 292 \\
\hline 22 & 244 & 296 \\
\hline 23 & 246 & 294 \\
\hline 24 & 226 & 309 \\
\hline 25 & 221 & 306 \\
\hline 26 & 231 & 317 \\
\hline 27 & 219 & 351 \\
\hline 28 & 224 & 295 \\
\hline 29 & 233 & 326 \\
\hline 30 & 233 & 303 \\
\hline Average & 234 & 318 \\
\hline \multicolumn{2}{|c|}{ Difference (b-a) } & 84 \\
\hline \multicolumn{2}{|c|}{ Percentage of difference (b-a) } & $26.4 \%$ \\
\hline
\end{tabular}

The comparison of the average pings delay of each test for both proposed solutions is shown in fig. 18. Based on table 5 and fig. 18, we can see that when we use the BGP community solution, the average ping delay is $84 \mathrm{~ms}$ (26.4\%) faster than the OSPF over GRE tunnel solution

In BGP Community solution, the ICMP-echo-request reply time is faster because the number of hops traversed by $\mathrm{PC} \mathrm{HO}-\mathrm{PC}-01$ to the Intranet server in the Regional
Data Center is less than when using OSPF over GRE Tunnel solution, IE 10 hops versus 14 hops.

\section{TCP Three-Way Handshake Time for HTTP}

In every test, we record the time when the client PC initiates a connection by sending a TCP syn packet [19] to the intranet server with destination port 80 (TCPs) and the time when the client PC transmit TCP ack packet to the intranet server (TCPa), in response to the TCP synack packet sent by the intranet server. The HTTP TCP three-way handshake time (TCPt) can be calculated by using the following formula.

$$
\mathrm{TCP} t=\mathrm{TCP} a-\mathrm{TCP} s
$$

The time required to complete a TCP three-way handshake for HTTP connection between the client PC and intranet server when we use both solutions after interDC link is disconnected, can be seen in table 6 .

Table 6. TCP Three-way Handshake Time for HTTP Connection

\begin{tabular}{|c|c|c|}
\hline Test & BGP Community (a) & $\begin{array}{c}\text { OSPF over } \\
\text { GRE Tunnel (b) }\end{array}$ \\
\hline 1 & 238.4 & 374.1 \\
\hline 2 & 242.4 & 538.3 \\
\hline 3 & 258.8 & 349.7 \\
\hline 4 & 238.2 & 355.8 \\
\hline 5 & 238.3 & 397.8 \\
\hline 6 & 228.4 & 263.9 \\
\hline 7 & 313.9 & 368.7 \\
\hline 8 & 199.2 & 377.7 \\
\hline 9 & 281.3 & 280.8 \\
\hline 10 & 252 & 296.3 \\
\hline 11 & 219.6 & 220.7 \\
\hline 12 & 252.4 & 319.1 \\
\hline 13 & 320.2 & 274.2 \\
\hline 14 & 187.7 & 382.2 \\
\hline 15 & 218.5 & 484.2 \\
\hline 16 & 201.4 & 293 \\
\hline 17 & 193.5 & 320.7 \\
\hline 18 & 232 & 369.1 \\
\hline 19 & 338.4 & 295.8 \\
\hline 20 & 176.1 & 388.6 \\
\hline 21 & 232 & 396.9 \\
\hline 22 & 231.8 & 328.2 \\
\hline 23 & 189.2 & 308.4 \\
\hline 24 & 183.2 & 231 \\
\hline 25 & 178.6 & 463 \\
\hline 26 & 230.8 & 330.4 \\
\hline 27 & 290.5 & 379.8 \\
\hline 28 & 221.8 & 328.9 \\
\hline 29 & 199.7 & 361.5 \\
\hline 30 & 196.4 & 254.6 \\
\hline Average & 232.8 & 344.4 \\
\hline \multicolumn{2}{|c|}{ Difference (b-a) } & 111.6 \\
\hline \multicolumn{2}{|c|}{ Percentage of Difference (b-a) } & $32.4 \%$ \\
\hline
\end{tabular}


The comparison graph of the length of time it takes to complete a TCP three-way handshake for HTTP connection on both of our proposed solutions is shown in fig. 19.

Based on table 6 and fig. 19, we can see that when we use the BGP community solution, the average TCP three- way handshake time for HTTP connection is $111.6 \mathrm{~ms}$ (32.4\%) faster than the OSPF over GRE tunnel solution. This is because of the number of hops traversed by $\mathrm{PC}$ HO-PC-01 to the Intranet server when using the BGP Community solution less than when using the OSPF over GRE Tunnel solution, which is 10 hops to 14 hops.

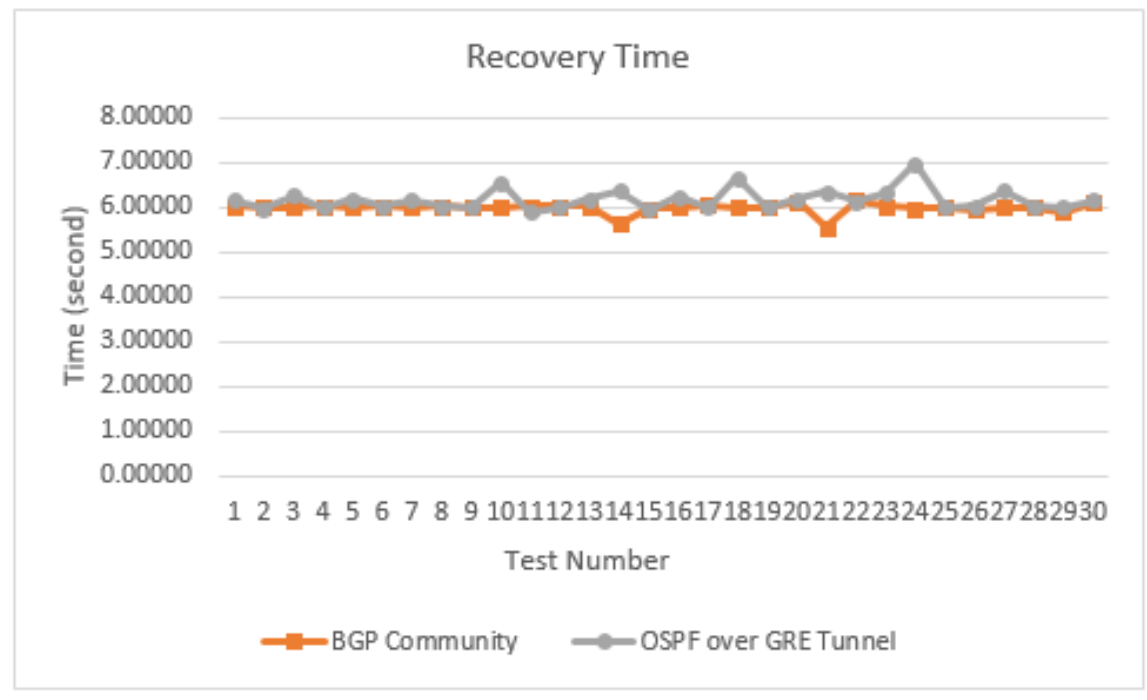

Fig.16. Recovery Time Graph

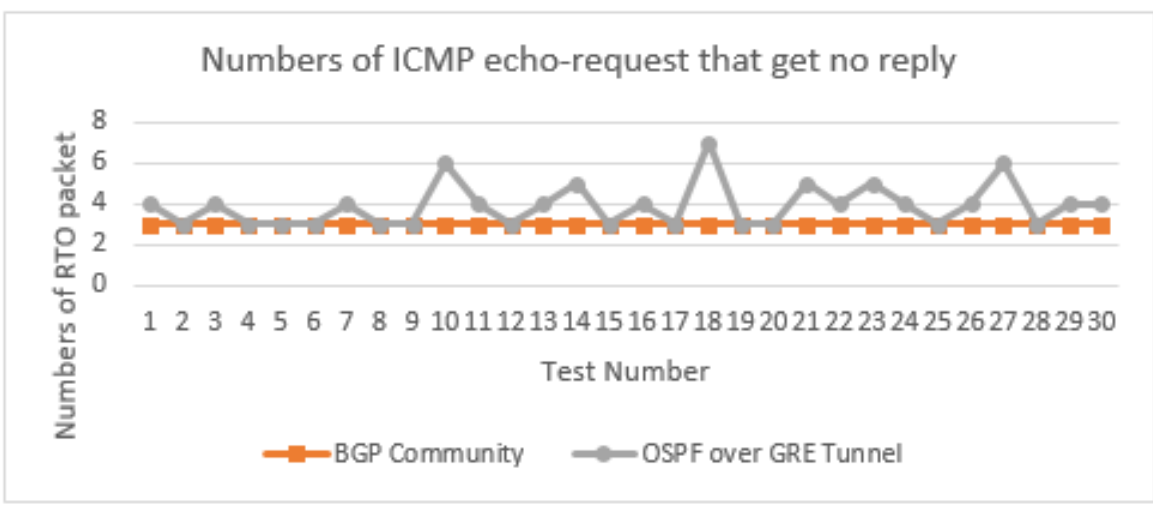

Fig.17. Number of ICMP Echo Packet That Does Not Get a Reply

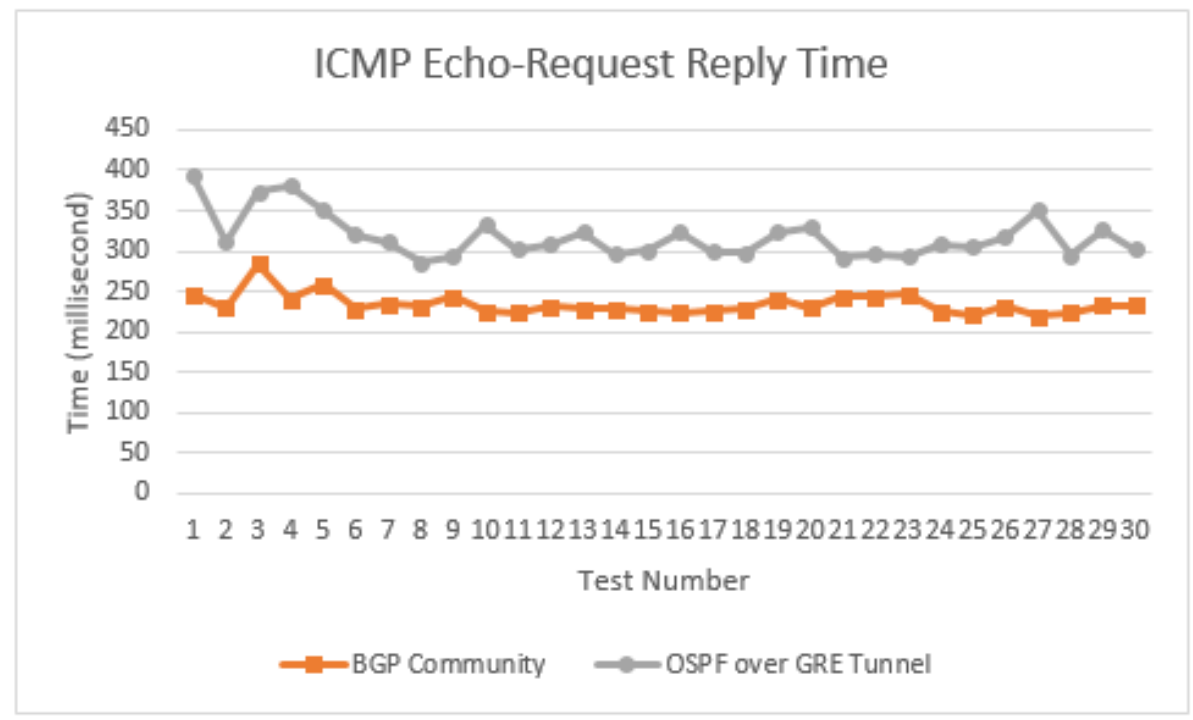

Fig.18. ICMP Echo-request Reply Time Graph 


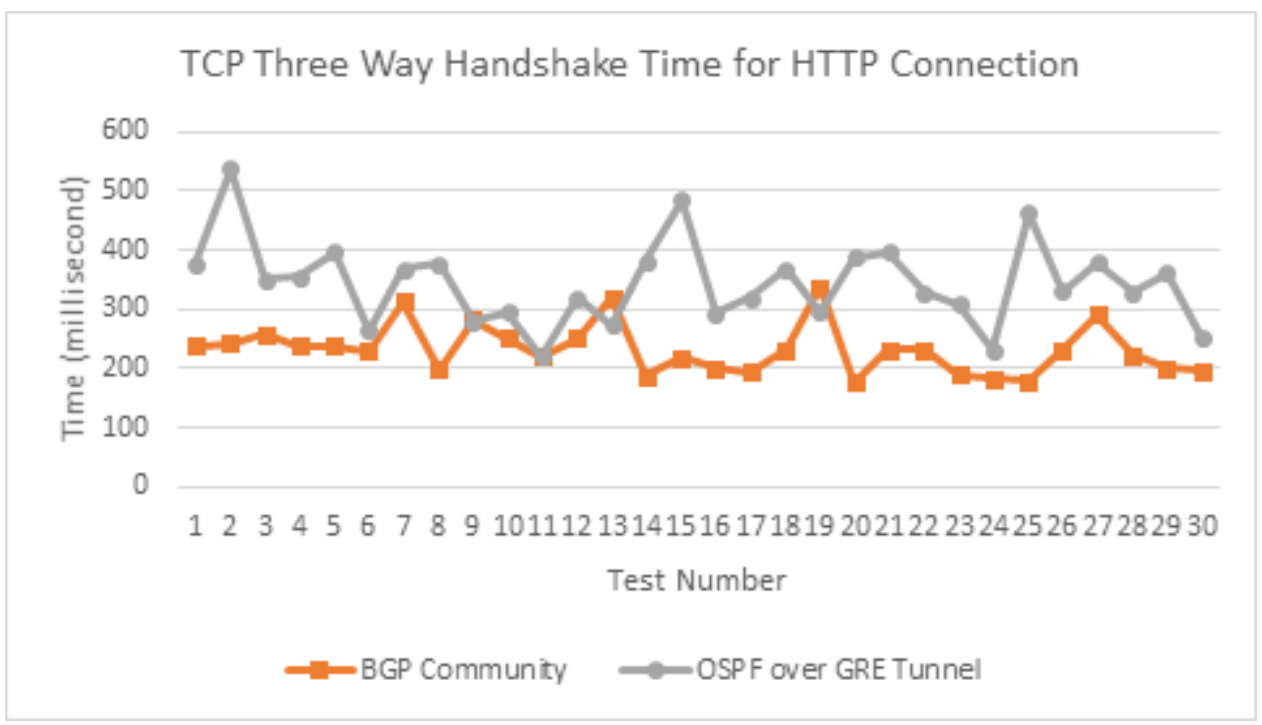

Fig.19. TCP three-way Handshake Time for HTTP Connection Graph

\section{CONCLUSION}

In the active-active data center that uses firewalls on each Data Center, asymmetric routing is very dangerous, because it will make the dissolution of communication between clients in the Data Center with the server on the other network. This happens because the initial traffic goes through a firewall located in one Data Center and its response traffic back through the firewall located in the other Data Center, so the response traffic will be dropped by the firewall. "Organization A" encountered this asymmetric routing problem.

We have simulated "Organization A"'s active-active Data Center network using the same IGP and EGP routing protocols, the same router operating system, the same routing policy and the same traffic flow. We also reproduce the same problem, ie dropped intranet web traffic when the inter-DC link is disconnected. Based on Analysis of "Organization A"'s network architecture and routing policy, we design, implement, verify and test the two solutions we offer using a simulator. Based on the 30 tests we conducted (that based on the 4 parameters we tested, IE recovery time, packet loss (the number of icmp echo-request packets that did not get replies), ping delay, and the time that required to complete the TCP three-way handshake for HTTP connection), we concluded that the BGP community solution is better than the OSPF over GRE Tunnel solution to be applied to "Organization A" networks, as well as other organizations that have similar network topology and routing policies.

\section{REFERENCES}

[1] Osunade, O (2012). A Packet Routing Model for Computer Networks. International Journal of Computer Network and Information Security. 4 (2012) 13-20.

[2] Bogdanoski, M., Shuminoski, T., \& Risteski, A. (2013). Analysis of the SYN Flood DoS Attack. International Journal of Computer Network and Information Security.8 (2013) 1-11.

[3] Durai, A. (2008). Asymmetric Routing and Firewalls.
Retrieved from Cisco Systems: https://www.cisco.com/web/services/news/ts_newsletter/t ech/chalktalk/archives/200903.html

[4] Piens, T. (2015). DotW: Issues with Asymmetric Routing. Retrieved from Palo Alto Networks: https://live.paloaltonetworks.com/t5/Featured-

Articles/DotW-Issues-with-Asymmetric-Routing/ta$\mathrm{p} / 65456$

[5] Magnani, D., Carvalho, I., Noronha, T. (2016). Robust Optimization for OSPF Routing. International Federation of Automatic Control. 49-12 (2016) 461-466.

[6] Periyasamy, P., Karthikeyan, E. (2013). Survey of Current Multipath Routing Protocols for Mobile AD Hoc Networks. International Journal of Computer Network and Information Security. 12 (2013) 68-79.

[7] Hiran, R., Carlsson, N., \& Shahmehri, N. (2017). Collaborative Framework for Protection Against Attack Targeting BGP and Edge Networks. Computer Networks, 122 (2017) 120-137.

[8] Cheng, P., Zhang, B., Massey, D., \& Zhang, L. (2010). Identifying BGP Routing Table Transfers. Computer Networks, 55 (2011) 636-649.

[9] Rekhter, Y., Li, T., (1995). RFC 1771: A Border Gateway Protocol 4 (BGP-4). Retrieved from Internet Engineering Task For19 (IETF): https://tools.ietf.org/html/rfc1771

[10] Cisco Systems (2016). BGP Best Path Selection Algorithm. Retrieved from Cisco Systems: https://www.cisco.com/c/en/us/support/docs/ip/bordergateway-protocol-bgp/13753-25.html

[11] Cisco Systems (2013). What is Administrative Distance?. Retrieved form Cisco Systems: https://www.cisco.com/c/en/us/support/docs/ip/bordergateway-protocol-bgp/15986-admin-distance.html

[12] Parkhurst, W. (2002). Cisco OSPF Command and Configuration Handbook.Cisco Press. Indianapolis, USA.

[13] Cisco Systems. (2013). Cisco IOS IP Routing: BGP Command Reference. Retrieved from Cisco Systems: https://www.cisco.com/c/en/us/td/docs/ios/iproute_bgp/co mmand/reference/irg_book/irg_bgp4.html

[14] Juniper Networks. hold-time (Protocols BGP). Retrieved on 20 February 2018 from Juniper Networks: https://www.juniper.net/documentation/en_US/junos/topic s/reference/configuration-statement/hold-time-editprotocols-bgp.html 
[15] Chandra, R., Traina P. (1996). RFC 1997: BGP Communities Attribute. Received from Internet $\begin{array}{llll}\text { Engineering Task Force (IETF): } & \end{array}$ https://tools.ietf.org/html/rfc1997

[16] Dommety, G. (2000). RFC 2890: Key and Sequence Number Extensions to GRE. Retrieved from Internet Engineering Task Force (IETF): https://tools.ietf.org/html/rfc2890

[17] Farinacci, D., Li, T., Hanks, S., Meyer, D., Traina, P. (2000). RFC 2784: Generic Routing Encapsulation (GRE) Retrieved from Internet Engineering Task Force (IETF): https://tools.ietf.org/html/rfc2784

[18] Postel, J. (1981). Internet Control Message Protocol. RFC 1972. Retrieved from Internet Engineering Task Force: https://tools.ietf.org/html/rfc792.

[19] Pandya, P. (2013). TCP/IP Packet Analysis. Computer and Information Security Handbook. (2013) 499-512.

\section{Authors' Profiles}

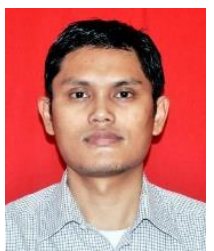

Irwan Piesessa received the Bachelor of Computer degrees from the High School of Informatics Management Muhamadiyah Jakarta, Indonesia, in 2005. Currently Irwan is studying in the postgraduate program in Informatics Engineering Department at Bina Nusantara University, Jakarta.

Previously, he worked in one of the largest telecommunication companies in Indonesia as a network security engineer. Now he works in a global telecommunications company as a senior network consultant. $\mathrm{He}$ has more than 15 years experience in Enterprise, Service
Provider, and Data Center network environment. He has a high interest in IP network management, network security and network design, planning and engineering.

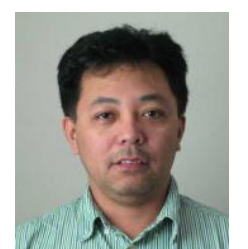

Benfano Soewito completed his bachelor level education in the F MIPA (since April 10, 2008 changed into the Faculty of Science and Technology) Department of Physics, Airlangga University, Surabaya, Indonesia. Then Benfano completed his master level education in the field of Computer Engineering at the Department of Electrical and Computer Engineering, Southern Illinois University, United States of America (USA) in 2004. Benfano Completed his doctoral level education in 2009 at the same faculty and university.

After completing his undergraduate level education, he worked at a company in Surabaya, which produces crystal oscillator as the head of engineering with the main task in the development of design specification, manufacturing technique and quality improvement of the crystal oscillator unit.

While completing his master and doctoral degree, he also worked as an assistant professor, research assistant, support computer specialist, and developed several websites inside the environment of Southern Illinois University, USA. After finishing his doctoral studies, he chose to pursue a career in the education field by joining Bakrie University. Since 2013, he has became a full-time lecturer at the Faculty of Graduate Programs at Bina Nusantara University, Jakarta. He has a high interest toward researches in computer science with a special interest in information technology as include Internet packet processing and scanning, router development as well as security and computer network.

How to cite this paper: Irwan Piesessa, Benfano Soewito,"Ensure Symmetrical Traffic Flow, to prevent the Dropping of Response Packet by the Firewall, on the Active-Active Data Centers", International Journal of Computer Network and Information Security(IJCNIS), Vol.10, No.6, pp.1-15, 2018.DOI: 10.5815/ijcnis.2018.06.01 